\title{
A Chemical Signature from Fast-rotating Low-metallicity Massive Stars: ROA 276 in $\omega$ Centauri
}

\author{
David Yong ${ }^{1}$, John E. Norris ${ }^{1}$, Gary S. Da Costa $^{1}$, Laura M. Stanford ${ }^{1}$, Amanda I. Karakas ${ }^{1,2}$, \\ Luke J. Shingles ${ }^{3}$, Raphael Hirschi ${ }^{4,5}$, and Marco Pignatari ${ }^{6,7,8}$ \\ ${ }^{1}$ Research School of Astronomy and Astrophysics, Australian National University, Canberra, ACT 2611, Australia; david.yong@anu.edu.au \\ ${ }^{2}$ Monash Centre for Astrophysics, School of Physics \& Astronomy, Monash University, Victoria 3800, Australia \\ ${ }^{3}$ Astrophysics Research Centre, School of Mathematics and Physics, Queen's University Belfast, Belfast BT7 1NN, UK \\ ${ }^{4}$ Astrophysics Group, Keele University, Staffordshire ST5 5BG, UK \\ ${ }^{5}$ Kavli Institute for the Physics and Mathematics of the Universe (WPI), The University of Tokyo, Kashiwa, Chiba 277-8583, Japan \\ ${ }^{6}$ E.A. Milne Centre for Astrophysics, Department of Physics \& Mathematics, University of Hull, HU6 7RX, UK \\ ${ }^{7}$ Konkoly Observatory, Hungarian Academy of Sciences, Budapest, Hungary \\ Received 2016 December 8; revised 2017 February 16; accepted 2017 February 17; published 2017 March 16
}

\begin{abstract}
We present a chemical abundance analysis of a metal-poor star, ROA 276, in the stellar system $\omega$ Centauri. We confirm that this star has an unusually high [Sr/Ba] abundance ratio. Additionally, ROA 276 exhibits remarkably high abundance ratios, $[\mathrm{X} / \mathrm{Fe}]$, for all elements from $\mathrm{Cu}$ to $\mathrm{Mo}$ along with normal abundance ratios for the elements from $\mathrm{Ba}$ to $\mathrm{Pb}$. The chemical abundance pattern of ROA 276, relative to a primordial $\omega$ Cen star ROA 46, is best fit by a fast-rotating low-metallicity massive stellar model of $20 M_{\odot},[\mathrm{Fe} / \mathrm{H}]=-1.8$, and an initial rotation 0.4 times the critical value; no other nucleosynthetic source can match the neutron-capture element distribution. ROA 276 arguably offers the most definitive proof to date that fast-rotating massive stars contributed to the production of heavy elements in the early universe.
\end{abstract}

Key words: globular clusters: individual ( $\omega$ Centauri) - stars: abundances - stars: Population II

Supporting material: machine-readable table

\section{Introduction}

Numerical simulations predict that low-metallicity stars that formed in the early universe were massive, compact, and rotated near their critical velocities where gravity is balanced by centrifugal forces (Bromm \& Larson 2004; Stacy et al. 2011). Nucleosynthesis in these fast-rotating lowmetallicity massive stars (hereafter spinstars) differs considerably from their non-rapidly rotating counterparts (Meynet et al. 2006; Hirschi 2007; Pignatari et al. 2008; Frischknecht et al. 2012, 2016; Maeder \& Meynet 2012). Since these massive stars have long since died, confirmation of their existence can be obtained by identifying their unique chemical signatures in the abundance patterns of subsequent generations of Milky Way stars (Frebel \& Norris 2015; Maeder et al. 2015).

One chemical signature of spinstars comes from nitrogen abundances in metal-poor halo stars, which require primary production (Spite et al. 2005). While spinstars can naturally achieve such nucleosynthesis, hydrogen ingestion in massive stars (Pignatari et al. 2015) and intermediate-mass and super asymptotic giant branch (AGB) stars (Karakas 2010; Doherty et al. 2014) may also be responsible for nitrogen production in the early universe.

Another possible observational signature of spinstars comes from neutron-capture elements. The scatter in $\mathrm{Sr}$ and $\mathrm{Ba}$ abundances in low-metallicity halo stars can be explained by spinstars (Cescutti et al. 2013), but measurements of other neutron-capture elements (e.g., Y, Zr, La), when available, are also compatible with massive AGB stars (Fishlock et al. 2014). Chiappini et al. (2011) reported unusually high abundances for

* This paper includes data gathered with the $6.5 \mathrm{~m}$ Magellan Telescopes located at Las Campanas Observatory, Chile.

${ }^{8}$ NuGrid collaboration http://www.nugridstars.org. the elements $\mathrm{Sr}, \mathrm{Y}, \mathrm{Ba}$, and $\mathrm{La}$ in the bulge globular cluster NGC 6522, consistent with yields from spinstars. Those measurements, however, have since been revised downward and could also be explained by AGB stars (Barbuy et al. 2014; Ness et al. 2014). The unmistakable signature among the neutron-capture elements from spinstars has yet to be seen within an individual star.

\section{Target Selection and Observations}

$\omega$ Centauri is the most massive star cluster in our Galaxy. In contrast to the majority of Milky Way globular clusters, $\omega$ Cen exhibits a number of peculiar features including a broad range in abundances for iron and slow neutron-capture process, or $s$ process, elements (Norris \& Da Costa 1995). The distribution and evolution of the $s$-process element abundances in $\omega$ Cen are consistent with a dominant contribution from 1.5 to $3 M_{\odot}$ AGB stars (Smith et al. 2000).

There are two stars in $\omega$ Cen, however, that exhibit peculiar abundance ratios of $\mathrm{Sr}$ and $\mathrm{Ba}$ (Stanford et al. 2006, 2010); the red giant ROA 276 with $V=12.37$ and the main-sequence star 2015448 with $V=18.22$. Both objects have high $\mathrm{Sr}$ and low $\mathrm{Ba}$ abundances, consistent with predictions of neutron-capture nucleosynthesis in spinstars (Frischknecht et al. 2012, 2016).

To further examine these unusual abundance patterns, we obtained a high-resolution optical spectrum for the red giant ROA 276 and a comparison star ROA $46(V=11.54)$ using the Magellan Inamori Kyocera Echelle spectrograph (Bernstein et al. 2003) at the $6.5 \mathrm{~m}$ Magellan Clay Telescope on 2007 June 22-23. Both stars have proper motions and radial velocities consistent with cluster membership (Bellini et al. 2009). The total exposure time was 10 minutes per target. We used the 0 ".5 slit to achieve a spectral resolution of $R=56,000$ and $R=44,000$ in the blue and red arms, respectively. 
Table 1

Stellar Parameters for the Program Stars

\begin{tabular}{lcccccc}
\hline \hline Star & $\begin{array}{l}T_{\text {eff }} \\
(\mathrm{K})\end{array}$ & $\begin{array}{c}\log g \\
(\mathrm{cgs})\end{array}$ & $\begin{array}{c}\xi_{t} \\
\left(\mathrm{~km} \mathrm{~s}^{-1}\right)\end{array}$ & $\begin{array}{c}{[\mathrm{Fe} / \mathrm{H}]} \\
(\mathrm{dex})\end{array}$ & $\begin{array}{c}T_{\text {eff }} \\
(\mathrm{K}) \\
\text { Photometric }\end{array}$ & $\begin{array}{c}\log g \\
(\mathrm{cgs})\end{array}$ \\
\hline ROA 276 & 4125 & 0.70 & 1.75 & -1.30 & 4130 & 0.79 \\
ROA 46 & 4075 & 0.20 & 2.40 & -1.72 & 4024 & 0.37 \\
\hline
\end{tabular}

One-dimensional, wavelength calibrated, continuum normalized spectra were produced from the raw spectra using IRAF ${ }^{9}$ and the MTOOLS ${ }^{10}$ package. The signal-to-noise ratio $(\mathrm{S} / \mathrm{N})$ for both stars was roughly 80 per pixel near $6000 \AA$ and 40 per pixel near $4500 \AA$. The spectra have approximately 3.5 pixels per resolution element.

\section{Stellar Parameters and Chemical Abundances}

The stellar parameters were determined from a traditional spectroscopic approach following the procedure outlined in Yong et al. (2014). Equivalent widths (EWs) were measured using routines in IRAF and DAOSPEC (Stetson \& Pancino 2008), and there was good agreement between the two approaches. Weak $(\mathrm{EW}<10 \mathrm{~m} \AA)$ and strong $(\mathrm{EW}>150 \mathrm{~m} \AA)$ lines were removed from the analysis. Abundances were derived using the $\mathrm{EW}$, one-dimensional local thermodynamic equilibrium (LTE) model atmospheres with $[\alpha / \mathrm{Fe}]=+0.4$ (Castelli \& Kurucz 2004), and the LTE stellar line analysis program MOOG (Sneden 1973). The version of MOOG that we used includes a proper treatment of Rayleigh scattering (Sobeck et al. 2011). The effective temperature $\left(T_{\text {eff }}\right)$, surface gravity $(\log g)$, and microturbulent velocity $\left(\xi_{t}\right)$, were obtained by enforcing excitation and ionization balance for Fe lines (see Table 1). The uncertainties in $T_{\text {eff }}, \log g$, and $\xi_{t}$ are $50 \mathrm{~K}, 0.2 \mathrm{dex}$, and $0.2 \mathrm{~km} \mathrm{~s}^{-1}$, respectively. The standard deviation for Fe I lines was 0.19 dex (ROA 276) and 0.16 dex (ROA 46), and we adopted an uncertainty in the model atmosphere of $[\mathrm{m} / \mathrm{H}]=0.2$ dex.

Stellar parameters can also be derived from a photometric approach. $T_{\text {eff }}$ can be estimated from color-temperature relations based upon the infrared flux method (Blackwell \& Shallis 1977; Ramírez \& Meléndez 2005). We used BVRIJHK photometry (Skrutskie et al. 2006; Bellini et al. 2009) and adopted a reddening of $E(B-V)=0.12$ (Harris 1996, 2010 edition). The surface gravity can be determined assuming the photometric $T_{\text {eff }}$, a distance modulus $(m-M)_{V}=13.94$ (Harris 1996, 2010 edition), bolometric corrections from Alonso et al. (1999), and a mass of $0.8 M_{\odot}$. $T_{\text {eff }}$ and $\log g$ obtained from the spectroscopic and photometric approaches are in good agreement when considering the estimated uncertainties (see Table 1).

Elemental abundances were derived using MOOG for individual lines based on the EW or from spectrum synthesis following Yong et al. (2014). Examples of synthetic spectra fits for representative lines of selected elements are given in Figure 1. Aside from the 4057.81 $\mathrm{Pb}$ I line, given the $\mathrm{S} / \mathrm{N}$ of the blue spectra, we analyzed lines redward of $4317.31 \AA$.

\footnotetext{
9 IRAF is distributed by the National Optical Astronomy Observatory, which is operated by the Association of Universities for Research in Astronomy (AURA) under a cooperative agreement with the National Science Foundation. ${ }_{10}$ www.lco.cl/telescopes-information/magellan/instruments/mike/iraftools/iraf-mtools-package
}

We present our line list, EWs, and abundance measurements in Table 2. Solar abundances were taken from Asplund et al. (2009) and the sources of the $g f$ values can be found in Table 2.

Uncertainties in chemical abundances were obtained by repeating the analysis and varying the stellar parameters, one at a time, by their uncertainties. These four error terms were added, in quadrature, to obtain the systematic uncertainty. We replaced the random error (s.e. $\log \epsilon)$ by $\max ($ s.e. $\log \epsilon$, $\left.0.20 / \sqrt{N_{\text {lines }}}\right)$, where the second term is what would be expected for a set of $N_{\text {lines }}$ with a dispersion of 0.20 dex. The total error was obtained by adding the random and systematic errors in quadrature. Chemical abundances and their errors are presented in Table 3.

\section{Results}

We measured abundances for 28 elements in both stars (see Figure 2). The comparison star ROA 46, with $[\mathrm{Fe} / \mathrm{H}]=-1.7$, belongs to the most metal-poor primordial population of $\omega \mathrm{Cen}$. This star has element abundance ratios relative to iron, $[\mathrm{X} / \mathrm{Fe}]$, that are typical for both field halo stars and $\omega$ Cen stars of comparable metallicity. For ROA 276 , with $[\mathrm{Fe} / \mathrm{H}]=-1.3$, the abundance ratios relative to iron for the elements from $\mathrm{Cu}$ to Mo are remarkably high and unusual, and we confirm the high $[\mathrm{Sr} / \mathrm{Ba}]$ ratio reported by Stanford et al. (2010). For other elements, the abundance ratios appear normal when compared to stars of similar metallicity.

In the context of the star-to-star light element abundance variations in globular clusters (Kraft 1994; Gratton et al. 2004), both ROA 276 and ROA 46 are "primordial" based on their O, $\mathrm{Na}$, and $\mathrm{Mg}$ abundances. That is, neither star has been affected by whatever process(es) produces the $\mathrm{O}-\mathrm{Na}$ anticorrelation in globular clusters (Charbonnel 2016).

The origin of the peculiar chemical composition of ROA 276 is revealed when we subtract the abundance pattern of the comparison star ROA 46 from ROA 276 (Figure 2, lower panel). The underlying assumptions in this approach are that (1) the comparison star represents the initial, or primordial, composition of $\omega$ Cen and (2) the peculiar star is produced by the addition of processed material onto the primordial composition. This methodology of examining relative abundance ratios has proved an extremely effective tool for identifying the source responsible for contaminating chemically peculiar objects (Roederer et al. 2011; Shingles et al. 2014). By considering relative abundance ratios rather than absolute abundances, systematic errors associated with a standard spectroscopic analysis largely cancel out (Meléndez et al. 2009).

\section{Discussion}

With the above assumptions in mind, we begin the discussion by noting that the $s$-process abundance distribution we see in stars depends upon the integrated exposure to neutrons (a quantity usually represented by $\tau$; Clayton 1968). The abundance pattern we see in Figure 2 is characteristic of a low value of $\tau$, that is, a small integrated neutron exposure, which means that elements beyond the first $s$-process peak are difficult to synthesize (e.g., Käppeler et al. 2011). A low value of $\tau$ is characteristic of the $s$-process operating in spinstars models (Frischknecht et al. 2016) and in intermediate-mass AGB models (e.g., Karakas et al. 2012), both of which release neutrons predominantly by the ${ }^{22} \mathrm{Ne}(\alpha, n)^{25} \mathrm{Mg}$ reaction. In 


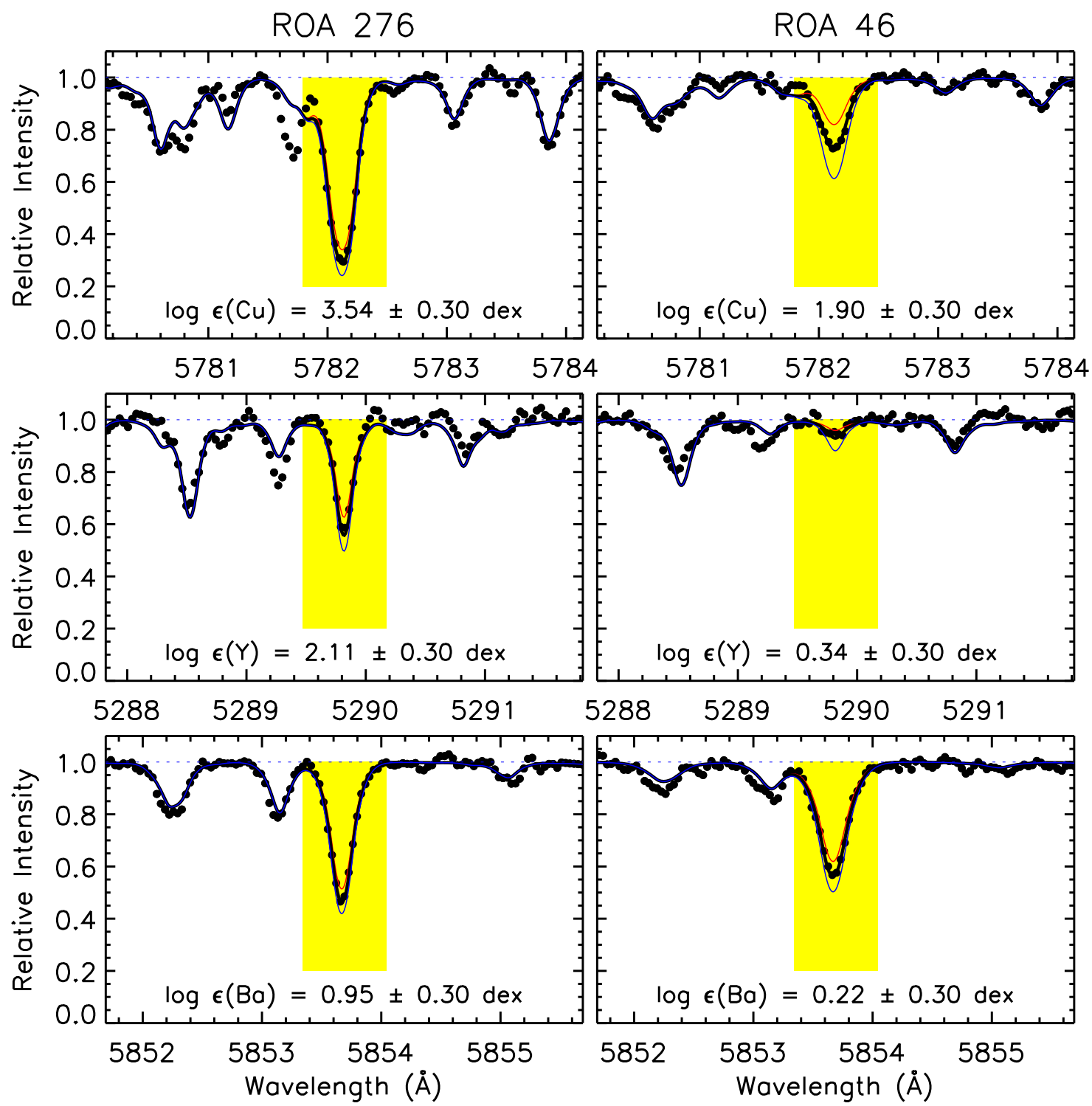

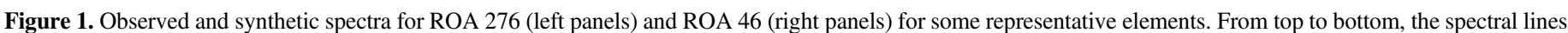



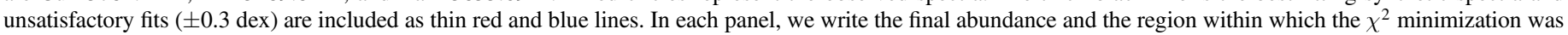
computed is indicated in yellow.

contrast, the $s$-process in low-mass AGB stars occurs via the ${ }^{13} \mathrm{C}(\alpha, n){ }^{16} \mathrm{O}$ reaction, which results in high neutron exposures overall and invariably results in high $\mathrm{Ba}$ and $\mathrm{Pb}$ abundances when compared to the first $s$-process peak (Busso et al. 1999; Karakas \& Lattanzio 2014). We now examine model predictions from intermediate-mass AGB stars and massive stars.

\subsection{Intermediate-mass AGB Stars}

Nucleosynthesis predictions from intermediate-mass AGB models of 5 and $7 M_{\odot}$ with $[\mathrm{Fe} / \mathrm{H}]=-1.2^{11}$ (Fishlock et al. 2014) offer an unsatisfactory fit to the data (Figure 3, upper panel). The rms (root mean square) scatter between observation and model is 0.38 and 0.43 dex for the 5 and $7 M_{\odot}$ models, respectively. A similarly poor fit to the data is obtained when using AGB yields from an independent group (Cristallo et al. 2011). In particular, nucleosynthesis occurring within

\footnotetext{
${ }^{11}$ These models adopt a scaled-solar composition.
}

non-rotating AGB models cannot simultaneously match the high abundances of $\mathrm{Cu}$ and $\mathrm{Zn}$ along with the high ratio of light $s$-process (e.g., $\mathrm{Rb}, \mathrm{Sr}, \mathrm{Y}, \mathrm{Zr}$ ) to heavy s-process elements (e.g., $\mathrm{Ba}, \mathrm{La}, \mathrm{Ce})$. For example, consider the following pairs of adjacent elements: (1) $\mathrm{Cu}$ and $\mathrm{Zn}$, (2) Rb and Sr, and (3) Ba and La. For a given pair of elements, we compute average values, e.g., $\langle\mathrm{Cu}, \mathrm{Zn}\rangle$, and ratios of these pairs, e.g., [ $\langle\mathrm{Cu}, \mathrm{Zn}\rangle /\langle\mathrm{Rb}, \mathrm{Sr}\rangle]$. For the 5 and $7 M_{\odot}$ AGB models by Fishlock et al. (2014), the ratios are $[\langle\mathrm{Cu}, \mathrm{Zn}\rangle /\langle\mathrm{Ba}, \mathrm{La}\rangle]=-0.04 \mathrm{dex}$ and $+0.05 \mathrm{dex}$, respectively, and these are a factor of 10 lower than the observed value of +1.08 dex. A similarly large discrepancy of $\sim 0.7 \mathrm{dex}$ between the observations and AGB calculations is found for the ratio $[\langle\mathrm{Rb}, \mathrm{Sr}\rangle /\langle\mathrm{Ba}, \mathrm{La}\rangle]$. Predictions from rotating AGB models are limited (Herwig et al. 2003; Siess et al. 2004; Piersanti et al. 2013) and do not include detailed $s$-process calculations for metal-poor intermediate-mass objects. Similarly, large grids of neutron-capture element yields for super-AGB models (rotating or non-rotating) are unavailable. 
Table 2

Line List, Equivalent Widths, and Abundances

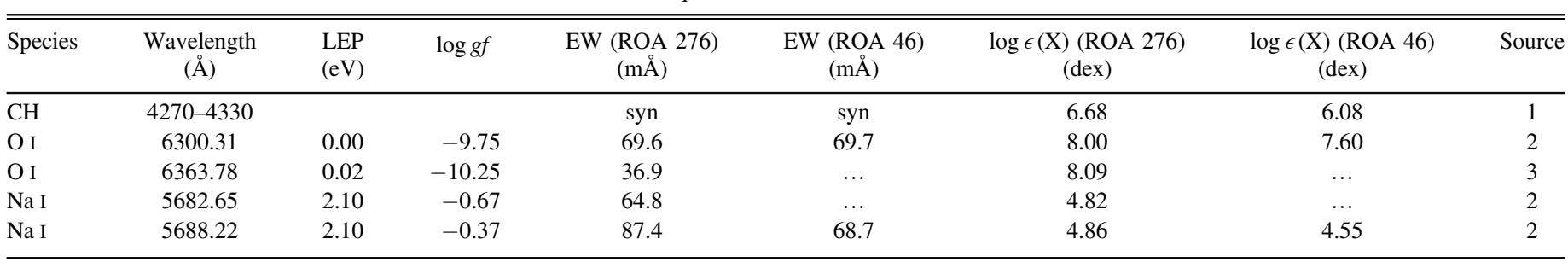

References. (1) Masseron et al. (2014), (2) Gratton et al. (2003) and references therein; (3) values as used in Yong et al. (2005) where the references include Kurucz \& Bell (1995), Prochaska et al. (2000), Den Hartog et al. (2003), Ivans et al. (2001), and Ramírez \& Cohen (2002), (4) Oxford group including Blackwell et al. (1979a, 1979b, 1980, 1986, 1995), (5) Kock \& Richter (1968), (6) Hannaford \& Lowe (1983), (7) Roederer \& Lawler (2012), (8) mean of lifetimes from Simsarian et al. (1998) and Volz \& Schmoranzer (1996) weighted according to uncertainties, via Morton (2000), (9) Wiese (2009), (10) Biémont et al. (2011), (11) Biemont et al. (1981), (12) Ljung et al. (2006), (13) Whaling \& Brault (1988), (14) Davidson et al. (1992) using hfs/IS from McWilliam (1998), (15) Lawler et al. (2001a), using hfs from Ivans et al. (2006), (16) Lawler et al. (2009), (17) Li et al. (2007), (18) Den Hartog et al. (2003), using hfs/IS from Roederer et al. (2008) when available; (19) Lawler et al. (2006), using hfs/IS from Roederer et al. (2008) when available; (20) Lawler et al. (2001b), using hfs/IS from Ivans et al. (2006), (21) Biémont et al. (2000), using hfs/IS from Roederer et al. (2012).

(This table is available in its entirety in machine-readable form.)

\subsection{Spinstars}

At low metallicity, fast rotation is an essential requirement to produce large quantities of neutron-capture elements in massive stars (Pignatari et al. 2008; Frischknecht et al. 2012, 2016). As noted above, the ${ }^{22} \mathrm{Ne}(\alpha, n)$ reaction is dominant in massive stars with a negligible contribution from the ${ }^{13} \mathrm{C}(\alpha, n)$ reaction (e.g., Baraffe et al. 1992; Nishimura et al. 2017), and the low neutron exposure limits the production of elements beyond the first $s$-process peak (Frischknecht et al. 2016). Nucleosynthesis predictions from spinstars by Frischknecht et al. $(2012,2016)$ provide an excellent fit to the relative abundance ratios for all 18 elements from $\mathrm{Cu}$ to $\mathrm{Pb}$ (Figure 3, lower panel). At metallicity $Z=10^{-3},[\mathrm{Fe} / \mathrm{H}]=-1.8,{ }^{12}$ which closely matches the comparison star ROA 46, the best fit is obtained from the $20 M_{\odot}$ model with an initial rotation rate 0.4 times the critical value. The rms is $0.25 \mathrm{dex}$, and this represents a superior fit when compared to the AGB models. The average measurement uncertainty is 0.24 dex, i.e., the rms can be attributed entirely to measurement errors. Additionally, the predicted and observed ratios for $[\langle\mathrm{Cu}, \mathrm{Zn}\rangle /\langle\mathrm{Ba}, \mathrm{La}\rangle]$ are +1.21 dex and +1.08 dex, respectively. Agreement is also obtained for $[\langle\mathrm{Rb}, \mathrm{Sr}\rangle /\langle\mathrm{Ba}, \mathrm{La}\rangle]$ with predicted and observed values of +1.70 dex and +1.42 dex, respectively. Therefore, the chemical abundance pattern of ROA 276, relative to the comparison star ROA 46, at present can be attributed to pollution from a spinstar. It is not clear, however, whether the spinstar polluted the gas cloud from which ROA 276 was formed or whether the pollution occurred via binary mass transfer. The principal result of this work is to provide clear observational support that the $s$-process in rapidly rotating massive stars was a relevant nucleosynthesis source in the early universe.

We note that the spinstar yields of Frischknecht et al. (2012, 2016) are pre-supernova yields. The supernova explosion does not significantly affect the pre-supernova neutron-capture element distribution (Tur et al. 2009), while major changes are expected for other elements, like $\mathrm{Fe}$ and other iron-group elements (Nomoto et al. 2013). The grid of fast-rotating massive star models that we tested covers a modest range in mass, metallicity, and rotation, and this grid will need to be

\footnotetext{
${ }^{12}$ These models adopt an $\alpha$ enhancement of $[\alpha / \mathrm{Fe}] \simeq+0.5$.
}

expanded in the future. We considered all the Frischknecht et al. $(2012,2016)$ models and adopted a threshold rms of 0.38 dex, which corresponds to the $5 M_{\odot}$ AGB model that we regarded as unsatisfactory. Three additional models satisfied this criterion (the rms values range from $0.26 \mathrm{dex}$ to $0.32 \mathrm{dex}$ ). These models are (1) $25 M_{\odot}$, initial rotation rate 0.4 times the critical value, and metallicity $Z=10^{-3}$, (2) same as (1) but with $40 M_{\odot}$, and (3) same as (2) but with metallicity $Z=10^{-5}$. We disregard the latter model because the metallicity, $[\mathrm{Fe} / \mathrm{H}]=-3.8$, is too low compared to the program stars. At present, the spinstar models that provide the best fits to the data have masses between 20 and $40 M_{\odot}$, a rotation rate of 0.4 times the critical value, and a metallicity of $Z=10^{-3}$.

Spinstars, however, are predicted to synthesize large quantities of the light elements $\mathrm{C}, \mathrm{N}$, and $\mathrm{O}$ (Meynet et al. 2006; Maeder et al. 2015). The best-fitting model predicts enhancements of $\Delta$ $[\mathrm{C} / \mathrm{Fe}]=+2.81, \Delta[\mathrm{C} / \mathrm{Sr}]=+0.72, \Delta[\mathrm{O} / \mathrm{Fe}]=+2.99$, and $\Delta[\mathrm{O} / \mathrm{Sr}]=+0.90$ and the observed ratios (ROA 276-ROA 46) are $+0.18,-1.51,+0.02$, and -1.57 , respectively. The $\mathrm{C}$ and $\mathrm{O}$ abundances in ROA 276 and ROA 46 are similar to each other and to metal-poor field giant stars (Stanford et al. 2010). Therefore, spinstar models predict differences in $\mathrm{C}$ and $\mathrm{O}$ between ROA 276 and ROA 46 that are at least two orders of magnitude larger than the observations. Either our proposed scenario of pollution from spinstars is incorrect, or the current models require refinement.

Although we dismissed AGB stars based on the neutroncapture element abundance distribution, here we consider the predicted yields for $[\mathrm{C} / \mathrm{Fe}],[\mathrm{O} / \mathrm{Fe}]$, and $[\mathrm{C} / \mathrm{Sr}]$ as we did for the spinstars. The relative abundance ratios (ROA 276-ROA 46) are $\Delta[\mathrm{C} / \mathrm{Fe}]=+0.18, \Delta[\mathrm{O} / \mathrm{Fe}]=+0.02$, and $\Delta[\mathrm{C} / \mathrm{Sr}]=-1.51$. The 5 and $7 M_{\odot}$ AGB models from Fishlock et al. (2014) with $[\mathrm{Fe} / \mathrm{H}]=-1.2$ predict $[\mathrm{C} / \mathrm{Fe}]=+1.01$ and +0.62 , $[\mathrm{O} / \mathrm{Fe}]=-0.20$ and -0.64 , and $[\mathrm{C} / \mathrm{Sr}]=-0.35$ and -0.21 , respectively. Therefore, while the AGB model predictions for $\mathrm{C}$ and $\mathrm{O}$ are not in major disagreement with the observations, the predicted $[\mathrm{C} / \mathrm{Sr}]$ ratios differ from the observations by at least an order of magnitude.

\subsection{ROA 46 as the Comparison Star}

The conclusions of this work depend on the decision to use ROA 46 as the reference star. In Figure 4, we plot various 
Table 3

Chemical Abundances of ROA 276 and the Comparison Star ROA 46

\begin{tabular}{|c|c|c|c|c|c|c|c|c|c|}
\hline Species & $\log \epsilon$ & $\sigma_{\log \epsilon}$ & $\begin{array}{l}{[\mathrm{Fe} / \mathrm{H}]} \\
\text { ROA } 276\end{array}$ & $\sigma_{[\mathrm{Fe} / \mathrm{H}]}$ & $\log \epsilon$ & $\sigma_{\log \epsilon}$ & $\begin{array}{l}{[\mathrm{Fe} / \mathrm{H}]} \\
\text { ROA } 46\end{array}$ & $\sigma_{[\mathrm{Fe} / \mathrm{H}]}$ & $\begin{array}{c}\Delta[\mathrm{Fe} / \mathrm{H}] \\
\text { ROA } 276-\mathrm{ROA} 46\end{array}$ \\
\hline $\mathrm{Fe} \mathrm{I}$ & 6.20 & 0.01 & -1.30 & 0.08 & 5.78 & 0.02 & -1.72 & 0.08 & 0.42 \\
\hline \multirow[t]{2}{*}{$\mathrm{Fe}$ II } & 6.21 & 0.04 & -1.29 & 0.15 & 5.80 & 0.03 & -1.70 & 0.13 & 0.41 \\
\hline & $\log \epsilon$ & $\sigma_{\log \epsilon}$ & ROA 276 & $\sigma_{[\mathrm{X} / \mathrm{Fe}]}$ & $\log \epsilon$ & $\sigma_{\log \epsilon}$ & ROA $46^{[\mathrm{X} / \mathrm{Fe}]}$ & $\sigma_{[\mathrm{X} / \mathrm{Fe}]}$ & $\begin{array}{c}\Delta[\mathrm{X} / \mathrm{Fe}] \\
\text { ROA } 276-\mathrm{ROA} 46\end{array}$ \\
\hline $\mathrm{C}(\mathrm{CH})$ & 6.68 & 0.20 & -0.45 & 0.24 & 6.08 & 0.20 & -0.63 & 0.24 & 0.18 \\
\hline O I & 8.04 & 0.05 & 0.65 & 0.18 & 7.60 & 0.20 & 0.63 & 0.22 & 0.02 \\
\hline $\mathrm{Na} I$ & 4.76 & 0.08 & -0.19 & 0.13 & 4.55 & 0.20 & 0.03 & 0.21 & -0.22 \\
\hline Mg I & 6.85 & 0.02 & 0.54 & 0.13 & 6.34 & 0.20 & 0.46 & 0.22 & 0.08 \\
\hline $\mathrm{Ca} \mathrm{I}$ & 5.54 & 0.03 & 0.50 & 0.14 & 5.00 & 0.04 & 0.38 & 0.13 & 0.12 \\
\hline Sc II & 1.48 & 0.06 & -0.37 & 0.14 & 1.49 & 0.06 & 0.06 & 0.13 & -0.43 \\
\hline Ti I & 4.12 & 0.02 & 0.47 & 0.13 & 3.70 & 0.03 & 0.47 & 0.13 & 0.00 \\
\hline Ti II & 4.21 & 0.05 & 0.56 & 0.14 & 3.69 & 0.03 & 0.46 & 0.12 & 0.10 \\
\hline Cr I & 4.45 & 0.06 & 0.11 & 0.11 & 4.03 & 0.12 & 0.10 & 0.16 & 0.01 \\
\hline $\mathrm{Cr}$ II & 4.35 & 0.12 & 0.01 & 0.18 & 4.12 & 0.07 & 0.20 & 0.17 & -0.19 \\
\hline Mn I & 3.71 & 0.03 & -0.42 & 0.10 & 3.36 & 0.06 & -0.35 & 0.10 & -0.07 \\
\hline Co I & 3.91 & 0.04 & 0.22 & 0.17 & 3.25 & 0.20 & -0.02 & 0.21 & 0.24 \\
\hline Ni I & 5.27 & 0.03 & 0.35 & 0.08 & 4.56 & 0.03 & 0.06 & 0.09 & 0.29 \\
\hline $\mathrm{Cu} \mathrm{I}$ & 3.56 & 0.02 & 0.67 & 0.17 & 1.96 & 0.06 & -0.51 & 0.16 & 1.18 \\
\hline $\mathrm{Zn} \mathrm{I}$ & 4.73 & 0.18 & 1.47 & 0.20 & 2.98 & 0.09 & 0.14 & 0.16 & 1.33 \\
\hline $\mathrm{Rb} \mathrm{I}$ & 3.15 & 0.03 & 1.93 & 0.17 & 1.22 & 0.20 & 0.42 & 0.23 & 1.51 \\
\hline Sr I & 2.90 & 0.03 & 1.32 & 0.17 & 0.78 & 0.20 & -0.37 & 0.21 & 1.69 \\
\hline Y II & 2.21 & 0.08 & 1.30 & 0.14 & 0.33 & 0.08 & -0.17 & 0.15 & 1.47 \\
\hline $\mathrm{Zr}$ I & 3.12 & 0.13 & 1.84 & 0.16 & 1.16 & 0.08 & 0.30 & 0.10 & 1.54 \\
\hline $\mathrm{Zr}$ II & 3.02 & 0.41 & 1.74 & 0.43 & 1.29 & 0.06 & 0.43 & 0.17 & 1.31 \\
\hline Mo I & 1.92 & 0.20 & 1.34 & 0.22 & 0.11 & 0.20 & -0.05 & 0.22 & 1.39 \\
\hline Ba II & 1.21 & 0.09 & 0.33 & 0.14 & 0.56 & 0.12 & 0.09 & 0.14 & 0.24 \\
\hline La II & -0.12 & 0.09 & 0.08 & 0.13 & -0.66 & 0.07 & -0.04 & 0.10 & 0.12 \\
\hline Ce II & 0.07 & 0.09 & -0.21 & 0.13 & -0.32 & 0.07 & -0.18 & 0.13 & -0.03 \\
\hline Pr II & -0.62 & 0.16 & -0.04 & 0.19 & -1.25 & 0.00 & -0.25 & 0.15 & 0.21 \\
\hline Nd II & 0.22 & 0.05 & 0.10 & 0.10 & -0.26 & 0.04 & 0.04 & 0.07 & 0.06 \\
\hline Sm II & -0.64 & 0.06 & -0.30 & 0.13 & -0.78 & 0.05 & -0.02 & 0.11 & -0.28 \\
\hline Eu II & -0.76 & 0.20 & 0.02 & 0.22 & -1.17 & 0.20 & 0.03 & 0.20 & -0.01 \\
\hline $\mathrm{Pb} \mathrm{I}$ & 0.70 & 0.20 & 0.25 & 0.23 & 0.45 & 0.20 & 0.42 & 0.24 & -0.17 \\
\hline
\end{tabular}

combinations of neutron-capture element abundances and compare ROA 276 and ROA 46 with a larger stellar sample from $\omega$ Cen (D'Orazi et al. 2011). Within the measurement uncertainties, ROA 46 is representative of the primordial population of $\omega$ Cen with low abundance ratios for the $s$ process elements. Thus, we may consider ROA 46 as a valid reference star.

\subsection{Alternative Nucleosynthetic Sites}

Another possibility worth considering is a truncated $s$-process operation involving the ${ }^{13} \mathrm{C}(\alpha, n){ }^{16} \mathrm{O}$ reaction in AGB stars. For the $2 M_{\odot}$ model with $[\mathrm{Fe} / \mathrm{H}]=-1.2$ from Fishlock et al. (2014), we examined the surface abundances after each thermal pulse. In contrast to ROA 276, the $\mathrm{Ba}$ and $\mathrm{Pb}$ abundances are high relative to the first $s$-process peak elements even after one to two thermal pulses and throughout the entire AGB phase. This model also predicts large $\mathrm{C}$ enhancements, which is also inconsistent with the observations. We therefore dismiss the AGB (and truncated AGB) scenario since it does not fit the neutron-capture element distribution and also fails to match the $\mathrm{C}$ and $\mathrm{O}$ abundances.

Beside the $s$-process discussed in this work for spinstars and AGB stars, other nucleosynthesis processes have been proposed as possible astrophysical sources of heavy elements (Thielemann et al. 2011).

The rapid neutron-capture process, or $r$-process, is not consistent with the abundance signature of ROA 276. The low $\mathrm{C}$ abundance and enhancements of the light $s$-process elements in ROA 276 are qualitatively similar to the observed abundance patterns in two halo stars (HD 122563 and HD 88609) and ET0097 in the Sculptor dwarf galaxy (Honda et al. 2006, 2007; Skúladóttir et al. 2015) and are attributed to the weak $r$-process (Arcones \& Montes 2011). The detailed abundance pattern of the neutron-capture elements in ROA 276 (or ROA 276-ROA 46), however, does not match HD 122563, HD 88609, or ET0097. Nucleosynthesis from the intermediate neutroncapture process or $i$-process (Cowan \& Rose 1977; Hampel et al. 2016; Jones et al. 2016; Denissenkov et al. 2017) is also inconsistent with the abundance signature of ROA 276.

While electron-capture supernovae are a potential source of neutron-capture elements, at present electron-capture supernovae models do not efficiently produce elements beyond $\mathrm{Zr}$ (Wanajo et al. 2011b). Such predictions are not consistent with ROA 276, where a strong production is observed up to Mo.

The alpha-rich freeze-out component ejected by high-energy core-collapse supernovae (CCSNe) does not seem to be compatible either when considering elemental ratios in the 


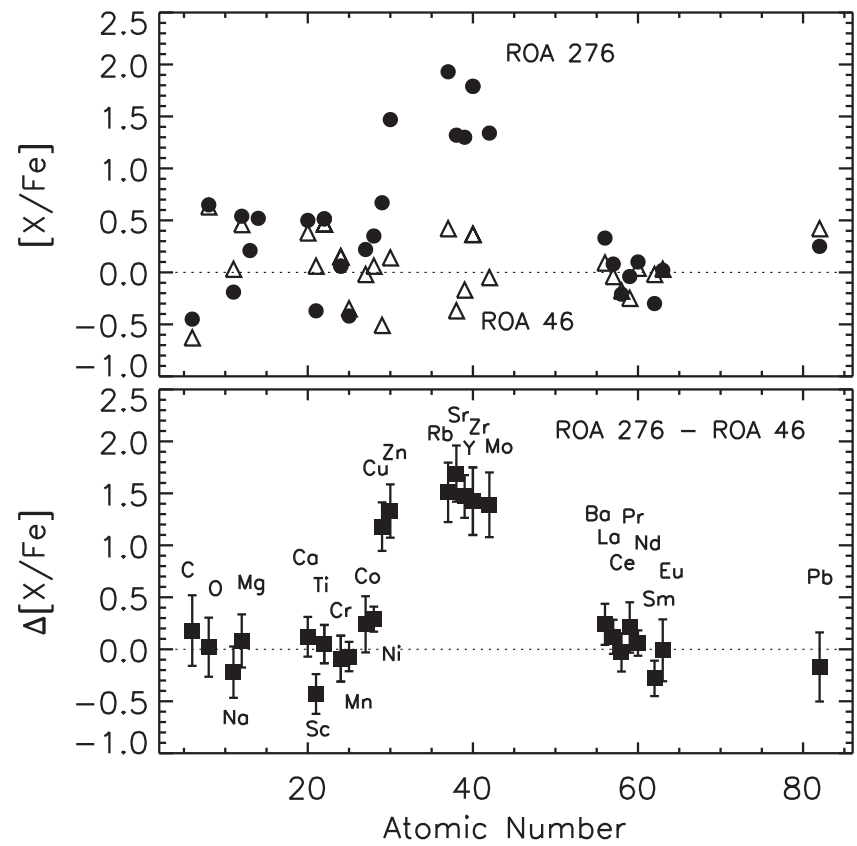

Figure 2. Element abundance ratios, $[\mathrm{X} / \mathrm{Fe}]$, as a function of atomic number (Top) Filled circles are ROA 276 and open triangles are the comparison star ROA 46. (Bottom) Relative abundance ratios in the sense ROA 276-ROA 46.



Figure 3. Comparison of relative abundance ratios (ROA 276-ROA 46) and model predictions as a function of atomic number. (Top) The dotted and solid lines are predictions from AGB models with $[\mathrm{Fe} / \mathrm{H}]=-1.2$ of 5 and $7 M_{\odot}$, respectively (Fishlock et al. 2014). (Bottom) The solid line is the prediction from a $20 M_{\odot}$ stellar model with $[\mathrm{Fe} / \mathrm{H}]=-1.8$ rotating at 0.4 times the critical value (Frischknecht et al. 2012, 2016).

$\mathrm{Rb}-\mathrm{Zr}$ region (Woosley \& Hoffman 1992). Furthermore, in alpha-rich freeze-out conditions, it is difficult to efficiently produce elements heavier than $\mathrm{Zr}$ (Pignatari et al. 2016).

Different types of neutrino-wind components in CCSNe can provide a large scatter of abundance patterns (Fröhlich et al. 2006; Kratz et al. 2008; Roberts et al. 2010; Arcones \&
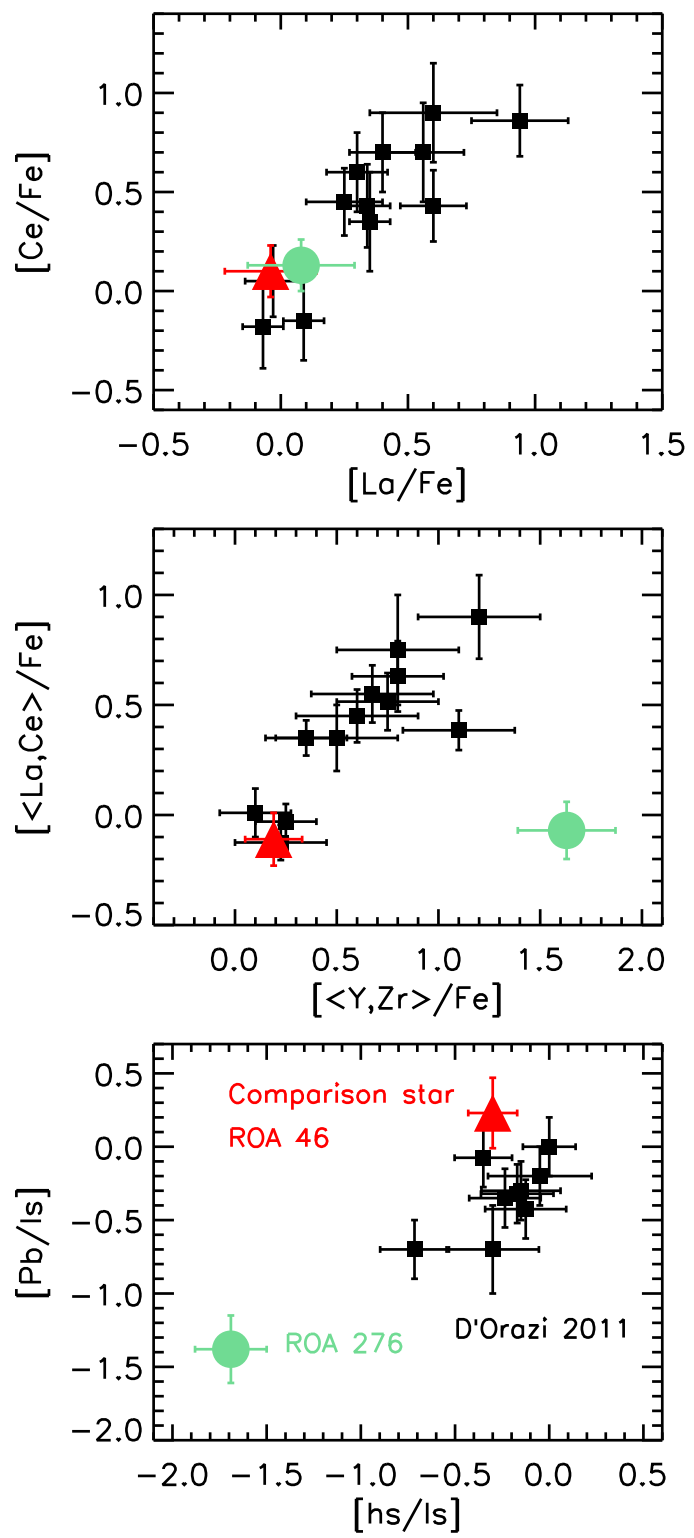

Figure 4. Neutron-capture element abundance ratios for ROA 276 (aqua circle), ROA 46 (red triangle), and $\omega$ Cen red giants (black squares from D'Orazi et al. 2011). $[\mathrm{Ce} / \mathrm{Fe}]$ vs. $[\mathrm{La} / \mathrm{Fe}]$ is shown in the upper panel, $[\langle\mathrm{La}, \mathrm{Ce}\rangle / \mathrm{Fe}]$ vs. $[\langle\mathrm{Y}, \mathrm{Zr}\rangle / \mathrm{Fe}]$ in the middle panel, and $[\mathrm{Pb} / \mathrm{ls}]$ vs. [hs $/ \mathrm{ls}]$ in the lower panel. "hs" is the average of $\mathrm{La}$ and $\mathrm{Ce}$, while "ls" is the average of $\mathrm{Y}$ and $\mathrm{Zr}$.

Montes 2011; Wanajo et al. 2011a), and a detailed study should be undertaken to check if there are reasonable stellar conditions that would produce material that would fit the ROA 276 abundance pattern between $\mathrm{Cu}$ and Mo. For instance, while high-entropy wind predictions seem to reproduce the observed pattern for $\mathrm{Sr}, \mathrm{Y}$, and $\mathrm{Zr}$, they underproduce $\mathrm{Rb}$ (Farouqi et al. 2009).

In summary, to our knowledge there are no nucleosynthetic sources, other than spinstars, that can match the neutroncapture element pattern in ROA 276. More complex astrophysical scenarios involving multiple sources could be invoked to explain the abundance pattern of ROA 276. While this would provide additional freedom to reproduce the observed data, any such scenario might be regarded as contrived and it is not obvious that we would find a combination of sources that 
could simultaneously fit the neutron-capture element pattern without large overabundances of $\mathrm{C}$ and other light elements.

\section{Conclusions}

We present a chemical abundance analysis of the red giant ROA 276 in $\omega$ Cen and a comparison red giant ROA 46. The neutron-capture element distribution of ROA 276, relative to ROA 46 , can be uniquely fit by nucleosynthesis predictions from a spinstar model with $20 M_{\odot},[\mathrm{Fe} / \mathrm{H}]=-1.8$, and an initial rotation rate 0.4 times the critical value.

ROA 276 was originally identified from a sample of 33 red giant branch stars in $\omega$ Cen (Stanford et al. 2010). Prior to this, examination of the $\mathrm{Sr}$ and $\mathrm{Ba}$ abundances in 392 mainsequence stars in $\omega$ Cen revealed only one object with high $\mathrm{Sr}$ and low Ba (Stanford et al. 2006). Among the $\sim 1000$ halo stars with $[\mathrm{Fe} / \mathrm{H}]<-1$ and $[\mathrm{Sr} / \mathrm{Ba}]$ measurements (Suda et al. 2008), only 13 have $[\mathrm{Sr} / \mathrm{Ba}]>+1.2$ and none exhibit the distinctive abundance pattern for the suite of elements from the Fe-peak through to $\mathrm{Pb}$ measured in ROA 276. Objects with chemical compositions similar to ROA 276 are rare. The predicted $[\mathrm{Sr} / \mathrm{Ba}]$ ratio from spinstars varies with mass and metallicity, and very high $[\mathrm{Sr} / \mathrm{Ba}]$ ratios only occur around metallicities $[\mathrm{Fe} / \mathrm{H}]=-2$ to -1 . ROA 276 (perhaps thanks to its metallicity and environment) provides a unique stellar laboratory to study neutron-capture nucleosynthesis in spinstars.

We thank the referee for helpful comments. D.Y., J.E.N., G.D.C., and A.I.K. acknowledge support from the Australian Research Council (grants DP0984924, FT110100475, DP120100475, DP120101237, FT140100554, and DP150103294). R.H. acknowledges support from EU-FP7ERC-2012-St Grant 306901. A.I.K. and R.H. acknowledge support from the World Premier International Research Center Initiative (WPI Initiative), MEXT, Japan. M.P. acknowledges significant support to NuGrid from NSF grants PHY 09-22648 (Joint Institute for Nuclear Astrophysics, JINA), NSF grant PHY-1430152 (JINA Center for the Evolution of the Elements), and EU MIRG-CT-2006-046520. M.P. acknowledges the support from the "Lendület-2014" Programme of the Hungarian Academy of Sciences (Hungary) and from SNF (Switzerland). Australian access to the Magellan Telescopes was supported through the Major National Research Facilities program of the Australian Federal Government. We acknowledge financial support from the Access to Major Research Facilities Program, under the International Science Linkages Program of the Australian Federal Government.

Facilities: Magellan:Clay (MIKE).

\section{References}

Alonso, A., Arribas, S., \& Martínez-Roger, C. 1999, A\&AS, 140, 261 Arcones, A., \& Montes, F. 2011, ApJ, 731, 5

Asplund, M., Grevesse, N., Sauval, A. J., \& Scott, P. 2009, ARA\&A, 47, 481 Baraffe, I., El Eid, M. F., \& Prantzos, N. 1992, A\&A, 258, 357

Barbuy, B., Chiappini, C., Cantelli, E., et al. 2014, A\&A, 570, A76

Bellini, A., Piotto, G., Bedin, L. R., et al. 2009, A\&A, 493, 959

Bernstein, R., Shectman, S. A., Gunnels, S. M., Mochnacki, S, \& Athey, A. E. 2003, Proc. SPIE, 4841, 1694

Biémont, E., Garnir, H. P., Palmeri, P., Li, Z. S., \& Svanberg, S. 2000, MNRAS, 312, 116

Biemont, E., Grevesse, N., Hannaford, P., \& Lowe, R. M. 1981, ApJ, 248, 867 Blackwell, D. E., Booth, A. J., Haddock, D. J., Petford, A. D., \& Leggett, S. K. 1986, MNRAS, 220, 549
Blackwell, D. E., Ibbetson, P. A., Petford, A. D., \& Shallis, M. J. 1979a, MNRAS, 186, 633

Blackwell, D. E., Lynas-Gray, A. E., \& Smith, G. 1995, A\&A, 296, 217

Blackwell, D. E., Petford, A. D., \& Shallis, M. J. 1979b, MNRAS, 186 657

Blackwell, D. E., Petford, A. D., Shallis, M. J., \& Simmons, G. J. 1980, MNRAS, 191, 445

Blackwell, D. E., \& Shallis, M. J. 1977, MNRAS, 180, 177

Biémont, É., Blagoev, K., Engström, L., et al. 2011, MNRAS, 414, 3350

Bromm, V., \& Larson, R. B. 2004, ARA\&A, 42, 79

Busso, M., Gallino, R., \& Wasserburg, G. J. 1999, ARA\&A, 37, 239

Castelli, F., \& Kurucz, R. L. 2004, (arXiv:astro-ph/0405087)

Cescutti, G., Chiappini, C., Hirschi, R., Meynet, G., \& Frischknecht, U. 2013, A\&A, 553, A51

Charbonnel, C. 2016, in EAS Publications Ser. 80, 177

Chiappini, C., Frischknecht, U., Meynet, G., et al. 2011, Natur, 472, 454

Clayton, D. D. 1968, Principles of Stellar Evolution and Nucleosynthesis (New York: McGraw-Hill)

Cowan, J. J., \& Rose, W. K. 1977, ApJ, 212, 149

Cristallo, S., Piersanti, L., Straniero, O., et al. 2011, ApJS, 197, 17

D’Orazi, V., Gratton, R. G., Pancino, E., et al. 2011, A\&A, 534, A29

Davidson, M. D., Snoek, L. C., Volten, H., \& Doenszelmann, A. 1992, A\&A 255,457

Den Hartog, E. A., Lawler, J. E., Sneden, C., \& Cowan, J. J. 2003, ApJS, 148,543

Denissenkov, P. A., Herwig, F., Battino, U., et al. 2017, ApJL, 834, L10

Doherty, C. L., Gil-Pons, P., Lau, H. H. B., et al. 2014, MNRAS, 441, 582

Farouqi, K., Kratz, K.-L., Mashonkina, L. I., et al. 2009, ApJL, 694, L49

Fishlock, C. K., Karakas, A. I., Lugaro, M., \& Yong, D. 2014, ApJ, 797 44

Frebel, A., \& Norris, J. E. 2015, ARA\&A, 53, 631

Frischknecht, U., Hirschi, R., Pignatari, M., et al. 2016, MNRAS, 456, 1803

Frischknecht, U., Hirschi, R., \& Thielemann, F.-K. 2012, A\&A, 538, L2

Fröhlich, C., Martínez-Pinedo, G., Liebendörfer, M., et al. 2006, PhRvL, 96, 142502

Gratton, R., Sneden, C., \& Carretta, E. 2004, ARA\&A, 42, 385

Gratton, R. G., Carretta, E., Claudi, R., Lucatello, S., \& Barbieri, M. 2003, A\&A, 404, 187

Hampel, M., Stancliffe, R. J., Lugaro, M., \& Meyer, B. S. 2016, ApJ, 831, 171

Hannaford, P., \& Lowe, R. M. 1983, OptEn, 22, 532

Harris, W. E. 1996, AJ, 112, 1487

Herwig, F., Langer, N., \& Lugaro, M. 2003, ApJ, 593, 1056

Hirschi, R. 2007, A\&A, 461, 571

Honda, S., Aoki, W., Ishimaru, Y., \& Wanajo, S. 2007, ApJ, 666, 1189

Honda, S., Aoki, W., Ishimaru, Y., Wanajo, S., \& Ryan, S. G. 2006, ApJ, 643,1180

Ivans, I. I., Kraft, R. P., Sneden, C., et al. 2001, AJ, 122, 1438

Ivans, I. I., Simmerer, J., Sneden, C., et al. 2006, ApJ, 645, 613

Jones, S., Ritter, C., Herwig, F., et al. 2016, MNRAS, 455, 3848

Käppeler, F., Gallino, R., Bisterzo, S., \& Aoki, W. 2011, RvMP, 83, 157

Karakas, A. I. 2010, MNRAS, 403, 1413

Karakas, A. I., García-Hernández, D. A., \& Lugaro, M. 2012, ApJ, 751, 8

Karakas, A. I., \& Lattanzio, J. C. 2014, PASA, 31, 30

Kock, M., \& Richter, J. 1968, ZAp, 69, 180

Kraft, R. P. 1994, PASP, 106, 553

Kratz, K.-L., Farouqi, K., Mashonkina, L. I., \& Pfeiffer, B. 2008, NewAR, 52,390

Kurucz, R. L., \& Bell, B. 1995, Atomic Line List (Cambridge: Smithsonian Astrophysical Observatory)

Lawler, J. E., Bonvallet, G., \& Sneden, C. 2001a, ApJ, 556, 452

Lawler, J. E., Den Hartog, E. A., Sneden, C., \& Cowan, J. J. 2006, ApJS, 162,227

Lawler, J. E., Sneden, C., Cowan, J. J., Ivans, I. I., \& Den Hartog, E. A. 2009, ApJS, 182, 51

Lawler, J. E., Wickliffe, M. E., den Hartog, E. A., \& Sneden, C. 2001b, ApJ, 563, 1075

Li, R., Chatelain, R., Holt, R. A., et al. 2007, PhyS, 76, 577

Ljung, G., Nilsson, H., Asplund, M., \& Johansson, S. 2006, A\&A, 456, 1181

Maeder, A., \& Meynet, G. 2012, RvMP, 84, 25

Maeder, A., Meynet, G., \& Chiappini, C. 2015, A\&A, 576, A56

Masseron, T., Plez, B., Van Eck, S., et al. 2014, A\&A, 571, A47

McWilliam, A. 1998, AJ, 115, 1640

Meléndez, J., Asplund, M., Gustafsson, B., \& Yong, D. 2009, ApJL, 704, L66

Meynet, G., Ekström, S., \& Maeder, A. 2006, A\&A, 447, 623

Morton, D. C. 2000, ApJS, 130, 403

Ness, M., Asplund, M., \& Casey, A. R. 2014, MNRAS, 445, 2994 
Nishimura, N., Hirschi, R., Rauscher, T., Murphy, A. S. J., \& Cescutti, G. 2017, MNRAS, submitted (arXiv:1701.00489)

Nomoto, K., Kobayashi, C., \& Tominaga, N. 2013, ARA\&A, 51, 457

Norris, J. E., \& Da Costa, G. S. 1995, ApJ, 447, 680

Piersanti, L., Cristallo, S., \& Straniero, O. 2013, ApJ, 774, 98

Pignatari, M., Gallino, R., Meynet, G., et al. 2008, ApJL, 687, L95

Pignatari, M., Herwig, F., Hirschi, R., et al. 2016, ApJS, 225, 24

Pignatari, M., Zinner, E., Hoppe, P., et al. 2015, ApJL, 808, L43

Prochaska, J. X., Naumov, S. O., Carney, B. W., McWilliam, A., \& Wolfe, A. M. 2000, AJ, 120, 2513

Ramírez, I., \& Meléndez, J. 2005, ApJ, 626, 465

Ramírez, S. V., \& Cohen, J. G. 2002, AJ, 123, 3277

Roberts, L. F., Woosley, S. E., \& Hoffman, R. D. 2010, ApJ, 722, 954

Roederer, I. U., \& Lawler, J. E. 2012, ApJ, 750, 76

Roederer, I. U., Lawler, J. E., Sneden, C., et al. 2008, ApJ, 675, 723

Roederer, I. U., Lawler, J. E., Sobeck, J. S., et al. 2012, ApJS, 203, 27

Roederer, I. U., Marino, A. F., \& Sneden, C. 2011, ApJ, 742, 37

Shingles, L. J., Karakas, A. I., Hirschi, R., et al. 2014, ApJ, 795, 34

Siess, L., Goriely, S., \& Langer, N. 2004, A\&A, 415, 1089

Simsarian, J. E., Orozco, L. A., Sprouse, G. D., \& Zhao, W. Z. 1998, PhRvA, 57,2448

Skrutskie, M. F., Cutri, R. M., Stiening, R., et al. 2006, AJ, 131, 1163

Skúladóttir, Á., Tolstoy, E., Salvadori, S., et al. 2015, A\&A, 574, A129
Smith, V. V., Suntzeff, N. B., Cunha, K., et al. 2000, AJ, 119, 1239

Sneden, C. 1973, ApJ, 184, 839

Sobeck, J. S., Kraft, R. P., Sneden, C., et al. 2011, AJ, 141, 175

Spite, M., Cayrel, R., Plez, B., et al. 2005, A\&A, 430, 655

Stacy, A., Bromm, V., \& Loeb, A. 2011, MNRAS, 413, 543

Stanford, L. M., Da Costa, G. S., \& Norris, J. E. 2010, ApJ, 714, 1001

Stanford, L. M., Da Costa, G. S., Norris, J. E., \& Cannon, R. D. 2006, ApJL, 653, L117

Stetson, P. B., \& Pancino, E. 2008, PASP, 120, 1332

Suda, T., Katsuta, Y., Yamada, S., et al. 2008, PASJ, 60, 1159

Thielemann, F.-K., Arcones, A., Käppeli, R., et al. 2011, PrPNP, 66 346

Tur, C., Heger, A., \& Austin, S. M. 2009, ApJ, 702, 1068

Volz, U., \& Schmoranzer, H. 1996, PhST, 65, 48

Wanajo, S., Janka, H.-T., \& Kubono, S. 2011a, ApJ, 729, 46

Wanajo, S., Janka, H.-T., \& Müller, B. 2011b, ApJL, 726, L15

Whaling, W., \& Brault, J. W. 1988, PhyS, 38, 707

Wiese, F. 2009, CRC Handbook of Chemistry and Physics (90th ed.; Boca Raton, FL: CRC Press), 10

Woosley, S. E., \& Hoffman, R. D. 1992, ApJ, 395, 202

Yong, D., Grundahl, F., Nissen, P. E., Jensen, H. R., \& Lambert, D. L. 2005, A\&A, 438, 875

Yong, D., Roederer, I. U., Grundahl, F., et al. 2014, MNRAS, 441, 3396 